Behaviour of metallic impurities at grain boundaries and dislocation clusters in multicrystalline silicon wafers deduced from contactless lifetime scan maps

This content has been downloaded from IOPscience. Please scroll down to see the full text. 2004 J. Phys.: Condens. Matter 16 S19

(http://iopscience.iop.org/0953-8984/16/2/003)

View the table of contents for this issue, or go to the journal homepage for more

Download details:

IP Address: 147.96.14.15

This content was downloaded on 26/09/2013 at 17:19

Please note that terms and conditions apply. 


\title{
Behaviour of metallic impurities at grain boundaries and dislocation clusters in multicrystalline silicon wafers deduced from contactless lifetime scan maps
}

\author{
P Hidalgo, O Palais ${ }^{1}$ and S Martinuzzi \\ Laboratoire TECSEN, UMR 6122, Universite de Marseille St Jerome case 231, \\ 13397 Marseille Cedex 20, France \\ E-mail: olivier.palais@univ.u-3mrs.fr
}

Received 31 July 2003, in final form 11 September 2003

Published 22 December 2003

Online at stacks.iop.org/JPhysCM/16/S19 (DOI: 10.1088/0953-8984/16/2/003)

\begin{abstract}
By means of a contactless microwave phase shift technique, the minority carrier lifetime and surface recombination velocity were measured in multicrystalline silicon wafers containing iron and chromium. The bulk lifetime can be deduced, leading to the determination of the concentration of interstitial iron $\left[\mathrm{Fe}_{\mathrm{i}}\right]$ associated with boron, after pair dissociation annealing at $210^{\circ} \mathrm{C}$. It is found that $\left[\mathrm{Fe}_{\mathrm{i}}\right]$ is higher in the vicinity of extended defects but decreases at the defects because the iron segregates irrespective of boron concentration and does not form pairs.
\end{abstract}

\section{Introduction}

The study of defect-defect and impurity-defect interactions in semiconductors, of which segregation of impurities is one of the possible consequences, has been and is still a hot subject of experimental and theoretical investigations [1]. It also became of crucial technological interest when it was recognized that shallow and deep impurities in silicon could be made electrically inactive by interaction with other defects or with extended defects. One issue of key technological importance is the urgent need to control the aggregation of impurities into microdefects. Multicrystalline silicon (mc-Si) used for solar cells is a well known example, as this material contains a lot of extended defects and impurities.

The lifetime $(\tau)$ of excess minority carriers is one of the most important parameters for the characterization of semiconductor wafers. When inhomogeneous samples, such as mc-Si wafers, are involved, it is also necessary to establish scan maps of this lifetime. Such maps could be deduced from minority carrier diffusion length $(L)$ maps, but it is also necessary to know the carrier diffusion coefficient as well as the spectral variation of the optical absorption coefficient. It is very often preferable to measure directly. Moreover, the techniques used

1 Author to whom any correspondence should be addressed. 
to determine $L$ need metal depositions to create contacts and collecting structures. These depositions have to be removed later for the manufacture of the devices and in some cases they can also contaminate the wafers. That is why contactless techniques involving microwaves are increasing in interest. The photoconductance decay (PCD) technique is the most commonly used but other techniques, like the contactless microwave phase shift ( $\mu \mathrm{W}$-PS), are increasing in importance. The $\mu \mathrm{W}$-PS technique uses the phase shift $(\phi)$ between a sinusoidal modulation of the excitation and the variation of the reflection power of microwaves.

However, since recombinations occur in the bulk as well as on both surfaces of the sample, the measured lifetime is in fact an effective lifetime $\left(\tau_{\text {eff }}\right)$, which depends on the bulk lifetime $\left(\tau_{\mathrm{b}}\right)$ and on the surface recombination velocity $S$, which itself depends strongly on the injection level. The effective lifetime value is given by

$$
\frac{1}{\tau_{\mathrm{eff}}}=\frac{1}{\tau_{\mathrm{b}}}+\frac{1}{\tau_{\mathrm{s}}}
$$

where $\tau_{\mathrm{s}}$ is the surface lifetime that depends directly on $S$. The knowledge as well as the control of $S$ by means of surface passivation techniques is indispensable to the determination of $\tau_{\mathrm{b}}$.

Normally, fast common diffusers like $\mathrm{Fe}$ and $\mathrm{Cr}$ are frequently involved in complexes with boron atoms in p-type materials and their recombination strength is modified, due to the difference in the capture cross-sections between the interstitial metal atoms and the complexes. Such complexes of iron and boron $(\mathrm{Fe}-\mathrm{B})$ or chromium and boron $(\mathrm{Cr}-\mathrm{B})$ are dissociated easily after a short annealing at temperatures in the range $200-250^{\circ} \mathrm{C}$, or after exposition to strong white light illumination. As a consequence, $\tau_{\mathrm{b}}$ varies and, from lifetime values before and after dissociation, we can deduce the concentration of these fast diffuser impurities included in the complexes. In the case of dissociation of $\mathrm{Fe}-\mathrm{B}$ pairs a decrease in the lifetime is observed but for $\mathrm{Cr}-\mathrm{B}$ pairs a higher value of $\tau$ is observed after dissociation.

In this work scan maps of minority carrier lifetime are obtained, by means of a $\mu \mathrm{W}$-PS technique, before and after thermal treatments at $210^{\circ} \mathrm{C}$ which allows us to determine distributions of $\mathrm{Fe}$ and $\mathrm{Cr}$ inside the grains and very close to the grain boundaries $(\mathrm{GBs})$ and dislocation clusters.

\section{Experimental details}

The investigation involved p-type mc-Si wafers, boron doped at about $10^{15} \mathrm{~cm}^{-3}$ and $270 \mu \mathrm{m}$ in thickness. Such wafers contain iron or chromium as the main metallic impurities. In order to reduce the concentration of impurities in samples, an external gettering process was carried out. Some wafers were iron rich and other were $\mathrm{Cr}$ rich. Before $\mu \mathrm{W}$-PS measurements the samples were cleaned with $\mathrm{HF}$ acid and deionised water to remove the oxide layer, dried in a flow of dry nitrogen and immediately mounted in the experimental setup.

Details of the experimental setup for $\mu \mathrm{W}$-PS measurements have been described elsewhere [2]. However, to obtain $\tau_{\mathrm{b}}$, it is necessary to control $S$ by means of surface passivation techniques. To avoid sample treatments at high temperatures, a very efficient technique, recently found, was used. Surface passivation was obtained by immersing the samples in an aqueous solution of iodine-polyvidone during the mapping of the lifetime. Such a passivation effect probably results from surface termination, as for the samples immersed in HF solution [3]. The passivation efficiency of the polyvidone solution is quite similar to that of iodine-ethanol [4]: there is no significant variation in the measured lifetime for $8 \mathrm{~h}$, at least.

From $\tau_{\text {eff }}$ it is possible determine the value of the bulk lifetime $\left(\tau_{\mathrm{b}}\right)$ and the value of the effective surface recombination velocity $S$ [5], (for passivated samples, $S$ is around $10^{3} \mathrm{~cm} \mathrm{~s}^{-1}$ ). 

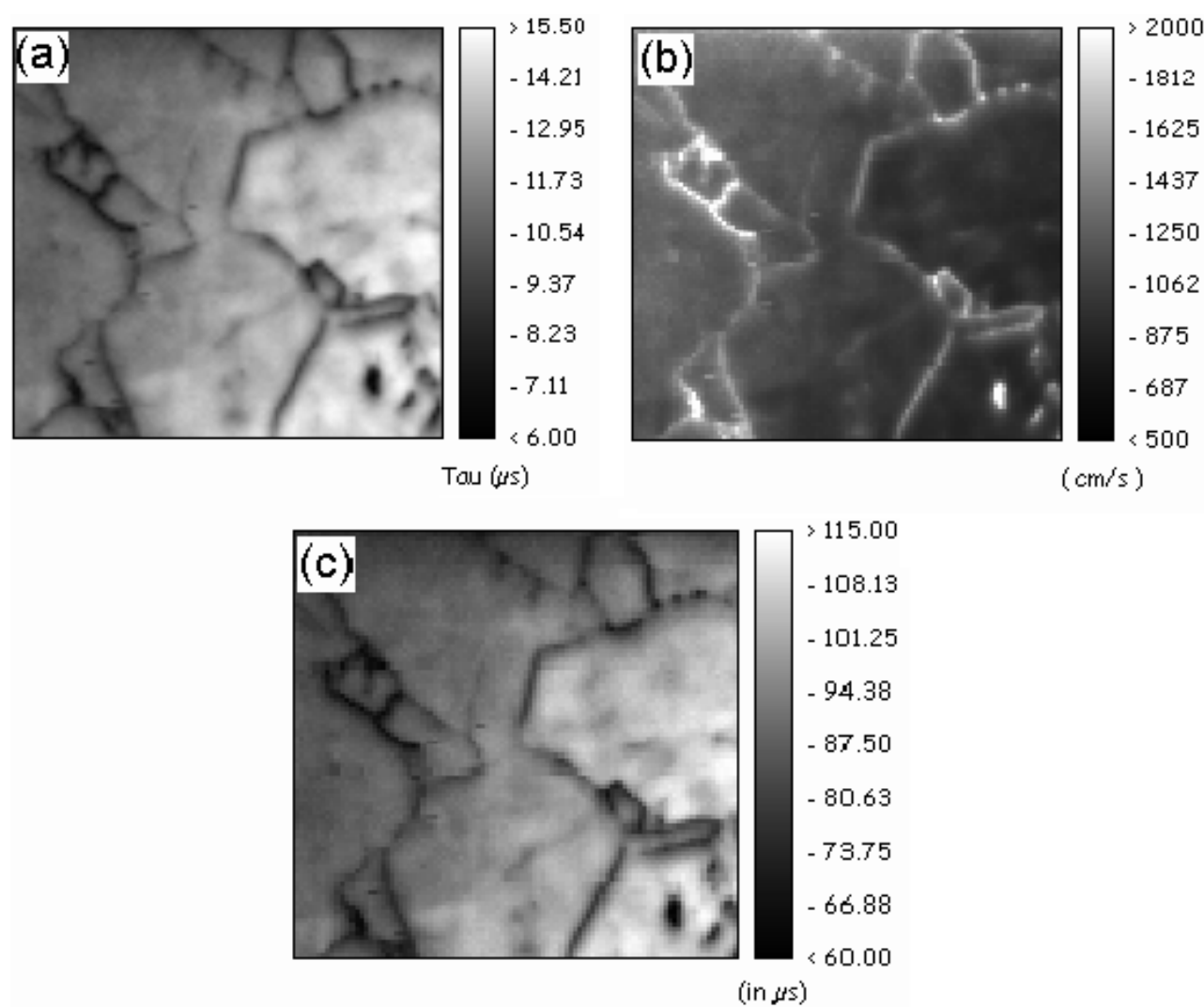

Figure 1. $10 \times 10 \mathrm{~mm}^{2}$ scan maps of (a) effective lifetime, (b) surface recombination velocity and (c) bulk lifetime before $\mathrm{Fe}-\mathrm{B}$ pairs dissociation annealing.

The method is based on the dependence of the phase shift $(\phi)$ with the modulation frequency of the exciting light. In this method of determining $\tau_{\mathrm{b}}$ and $S$ from data measured at different frequencies, it is assumed that the $S$ values for the two surfaces are the same.

According to the Shockley-Read-Hall (SRH) theory, the concentration of interstitial metallic atoms $\left(\mathrm{M}_{\mathrm{i}}\right)$ can be deduced from the expression [6]

$$
\left[\mathrm{M}_{\mathrm{i}}\right]=\kappa\left(\frac{1}{\tau_{\mathrm{after}}}-\frac{1}{\tau_{\text {before }}}\right)
$$

where $\kappa$ depends on the injection level (the relationship between excess carrier density and dopant concentration) and on the impurity type.

\section{Results}

From $\tau_{\text {eff }}$ scan maps measured at two different frequencies, it is possible to obtain the map of $\tau_{\mathrm{b}}$ and of $S$ before any thermal treatment, as shown in figure 1 . After annealing at $210^{\circ} \mathrm{C}$ for $10 \mathrm{~min}$, the sample is quickly immersed in deionised water at $0{ }^{\circ} \mathrm{C}$. The dissociation of complexes gives rise to a decrease of $\tau_{\text {eff }}$, and consequently of $\tau_{\mathrm{b}}$, in the homogeneous part of the grains, while at extended defects the variation of $\tau_{\text {eff }}$ is less marked, as shown by figure 2 (the scan map of $S$ is not shown because it is not modified). 

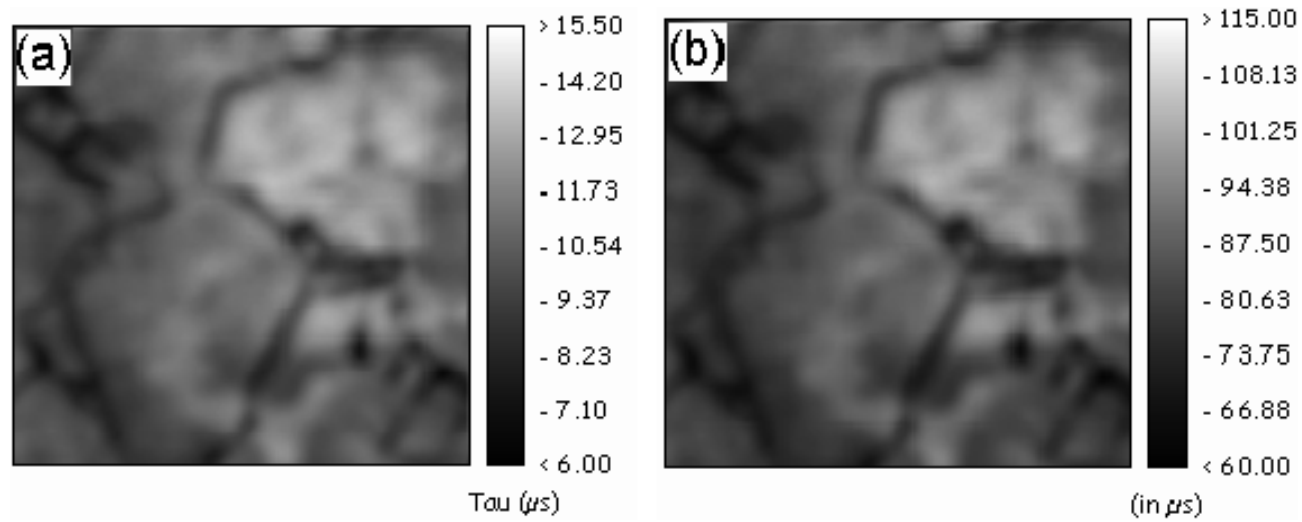

Figure 2. $10 \times 10 \mathrm{~mm}^{2}$ scan maps of (a) effective lifetime and (b) bulk lifetime after Fe-B pair dissociation annealing.

(a)

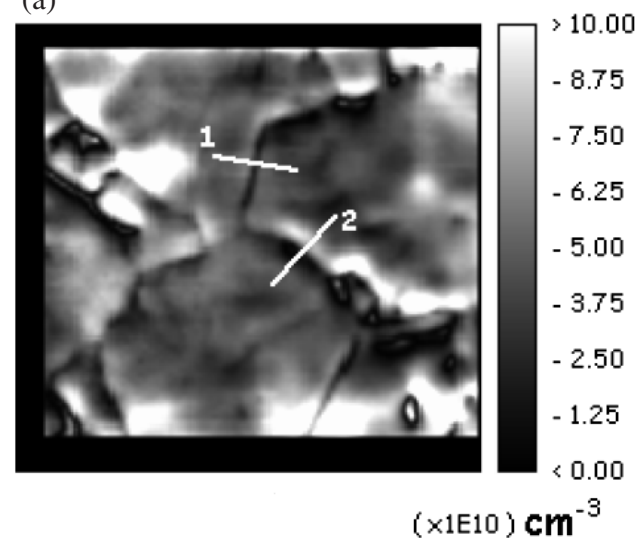

(b)

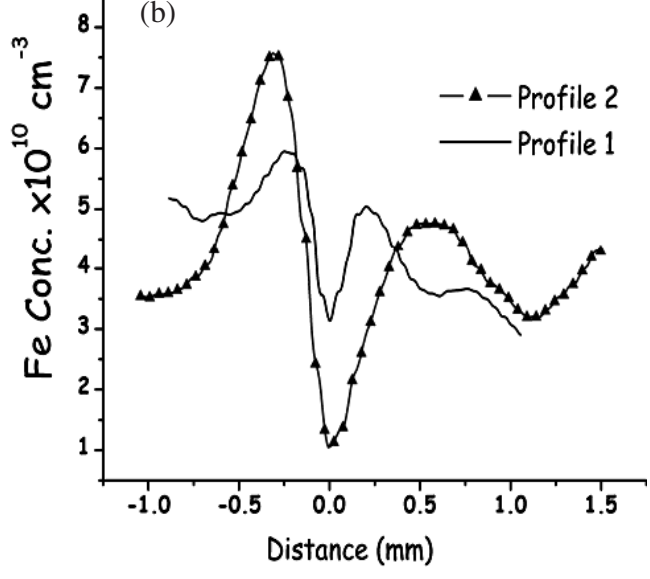

Figure 3. (a) $10 \times 10 \mathrm{~mm}^{2}$ scan map of interstitial iron concentration and (b) iron profile concentration across GBs 1 and 2 of (a).

Now, using expression (2) and [7] to obtain the $\kappa$ value for Fe, it is possible to determine the iron concentration in the sample. From the $\tau_{\mathrm{b}}$ scan maps, the iron concentration map can be deduced with the high spatial resolution of $50 \mu \mathrm{m}$, as shown in figure 3(a). It is clear that the iron concentration is higher close to GBs and extended defects (like dislocation clusters), due to an accumulation of iron around these defects. However at the defects, we detect a lower concentration of iron $\left(\simeq 10^{10} \mathrm{~cm}^{-3}\right)$, as shown by the profiles of figure $3(\mathrm{~b})$, probably because $\mathrm{Fe}$ is not associated with boron but with other imperfections contained in the GBs and dislocations.

Returning to the lifetime scan map of figures 1 and 2, we can obtain the $\tau_{\text {eff }}$ distribution, shown in figure $4(\mathrm{a})$, before and after the annealing at $210^{\circ} \mathrm{C}$. It appears that, before annealing there are two peaks, a broad one around $11.2 \mu$ s and another one, lower in intensity, around $13.5 \mu \mathrm{s}$. After annealing two new peaks appear, one at $9.5 \mu \mathrm{s}$ and another one at $10.2 \mu$ s related to the $\mathrm{Fe}-\mathrm{B}$ dissociation, which are associated with the GBs and extended defect vicinities, while the peak at $13.5 \mu$ s disappears. Indeed, if a given value of $\tau_{\text {eff }}$ is chosen in the maps of figures 1 and 2, it is possible to localize the regions of the sample where $\tau_{\text {eff }}=9.5 \mu \mathrm{s}$. 

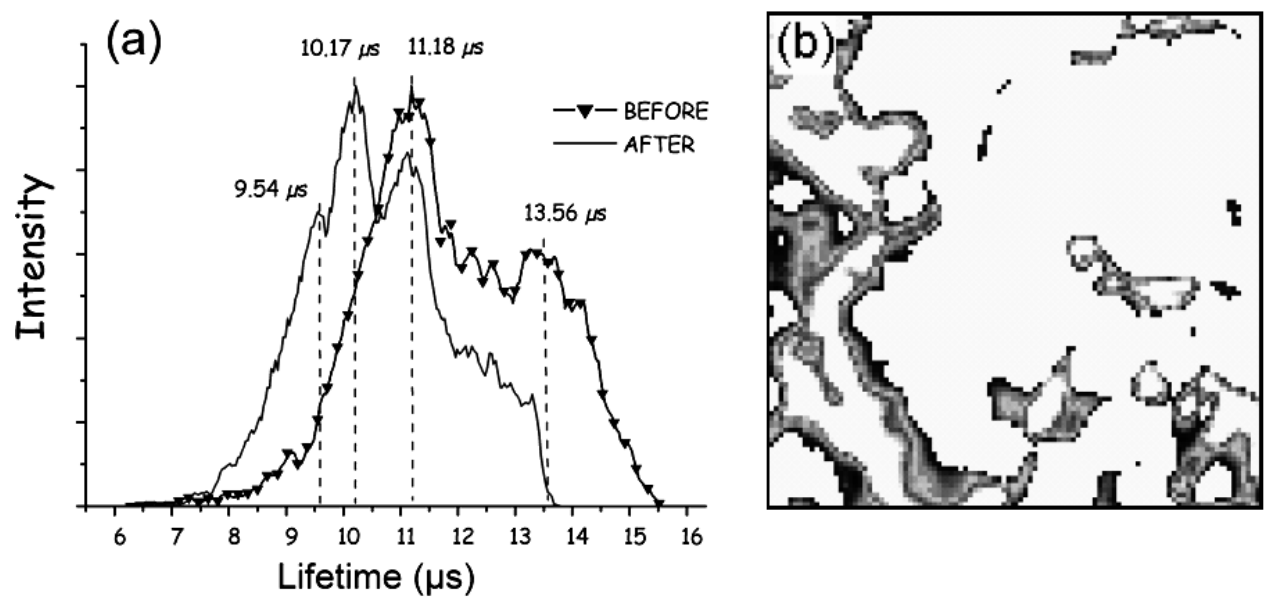

Figure 4. (a) Effective lifetime distribution in the investigated sample before and after dissociation annealing. (b) Localization of the regions where $\tau_{\text {eff }}=9.54 \mu$ s after dissociation annealing.

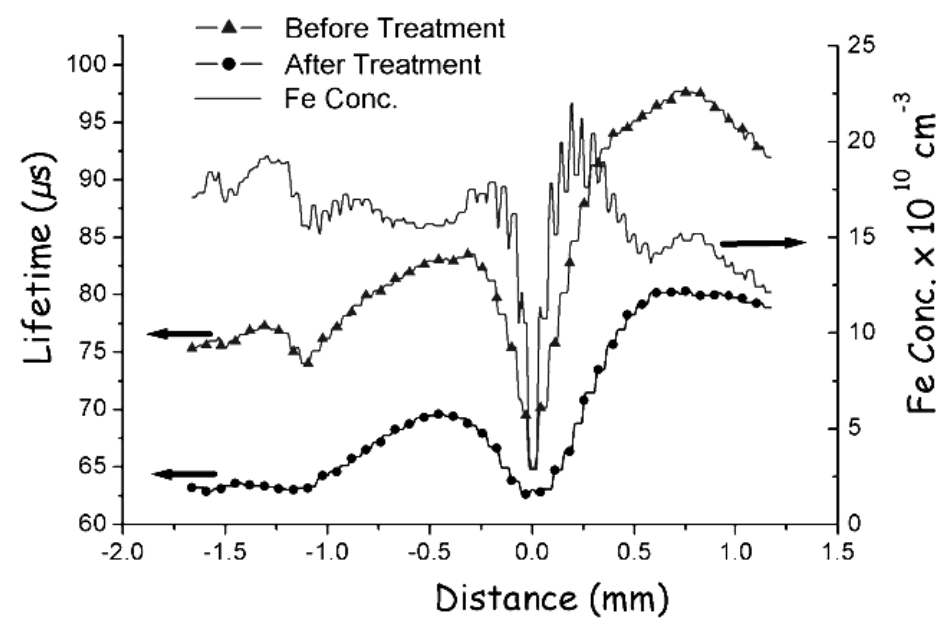

Figure 5. Bulk lifetime before and after dissociation annealing, and iron concentration profile across a GB.

As shown by figure 4(b), such regions are those containing a high density of dislocations and GBs. The peak at $11.2 \mu$ s remains with more or less the same intensity.

Lifetime and interstitial iron concentration profiles across a GB can be obtained by our technique, and an example is shown in figure 5. It appears that $\left[\mathrm{Fe}_{\mathrm{i}}\right]$ increases close to the defect but decreases at the defect. This could be due to iron segregation by the defect which increases $\left[\mathrm{Fe}_{\mathrm{i}}\right]$ in its vicinity; however at the GB itself, iron atoms are trapped irrespective of the boron concentration and, as they do not form complexes with boron, they cannot be detected.

In chromium rich samples the results are very similar except that $\tau$ increases after the dissociation annealing. Indeed it is found that $\left[\mathrm{Cr}_{\mathrm{i}}\right]$ atoms tend to be accumulated close to extended defects, while at the defects only few $\mathrm{Cr}$ atoms are detected, for the same reason. $\left[\mathrm{Cr}_{\mathrm{i}}\right]$ is certainly trapped but by dangling bonds or by other impurities. 


\section{Conclusions}

The $\mu \mathrm{W}$-PS technique leads to the determination of the bulk lifetime $\left(\tau_{\mathrm{b}}\right)$ of mc-Si. From the variation of $\tau_{\mathrm{b}}$ before and after annealing at $210^{\circ} \mathrm{C}$, the interstitial iron concentration produced by the $\mathrm{Fe}-\mathrm{B}$ pair dissociation annealing can be evaluated. Scan maps of $\left[\mathrm{Fe}_{\mathrm{i}}\right]$ show that this concentration is higher close to extended defects, while at the extended defects the measured concentration is found to be lower, due to the association of iron with defects contained in the GBs and dislocations rather than with boron.

\section{Acknowledgments}

This work was sponsored by CNRS/ADEME (France), by Conseils Regional (PACA) and General (13)-France and by Secretaria General Educacion y Universidades (Spain).

\section{References}

[1] Aucouturier M 1985 Polycrystalline Semiconductors: Physical Properties and Applications (Springer Series in Solid State Sciences vol 57) ed G Harbeke (Berlin: Springer) pp 47-74

[2] Palais O, Gervais J, Yakimov E and Martinuzzi S 2000 Eur. Phys. J. Appl. 10157

[3] Yablonovitch E and Gmitter T 1986 Appl. Phys. Lett. 49587

[4] Schroder D K 1990 Semiconductor Material and Device Characterization (New York: Wiley-Interscience) p 457

[5] Palais O and Arcari A 2003 J. Appl. Phys. 934686

[6] Lagowski J, Edelman P, Dexter M and Henley W 1992 Semicond. Sci. Technol. A 7185

[7] Palais O, Yakimov E and Martinuzzi S 2002 Mater. Sci. Eng. B 91/92 216 